\title{
Line Tunable Ultraviolet Laser
}

\author{
Brian M. Walsh and Norman P. Barnes \\ NASA Langley Research Center, Hampton, VA 23681 \\ Phone: (757)-864-7112, fax: (757) 864-8828, e-mailBrian.M.Walsh@nasa.gov
}

\begin{abstract}
An ultraviolet laser is demonstrated using a dual wavelength Nd:YAG oscillator, sum frequency and second harmonic process. Synchronous pulses at 1.052 and 1.319 micrometers are amplified, mixed and subsequently doubled, producing pulses at 0.293 micrometers.

(c)2004 Optical Society of America

OCIS codes: (140.3530) Lasers, neodymium; (140.3600) Lasers, tunable; (140.3610) Lasers, ultraviolet
\end{abstract}

\section{Introduction}

A laser in the ultraviolet is useful for remote sensing on the Earth. Range resolved measurements of ozone $\left(\mathrm{O}_{3}\right)$ and sulfur dioxide $\left(\mathrm{SO}_{2}\right)$ can be obtained using lidar techniques and line tunable lasers operating near $0.3 \mu \mathrm{m}$. Ozone is of interest because of its importance in solar ultraviolet shielding in the stratosphere. Sulfur dioxide is of interest because of smog pollution in the troposphere, and is a precursor to acid rain.

The dual wavelength laser oscillator, referred to as the STOP light laser [1], produces Synchronous Tunable Optical Pulses using Q-switched pulses from a single Nd:YAG oscillator operating on transitions between the ${ }^{4} \mathrm{~F}_{3 / 2}$ manifold to the ${ }^{4} \mathrm{I}_{11 / 2}$ and ${ }^{4} \mathrm{I}_{13 / 2}$ manifolds. Using an angle tuned BBO crystal, the second harmonic as well as the sum frequency of the 1.052 and $1.319 \mu \mathrm{m}$ pulses were demonstrated. Outputs were obtained at 0.526, 0.585, and $0.660 \mu \mathrm{m}$; green, orange, and red reminiscent of a STOP light. Second harmonic generation of pulses at $0.585 \mu \mathrm{m}$ produced ultraviolet radiation at $0.293 \mu \mathrm{m}$

\section{Experimental}

A dispersive resonator using a set of 4 quartz prisms provides common optical elements for collinear pulse generation as well as a tuning mechanism, shown in Figure 1. Both the 1.052 and $1.319 \mu \mathrm{m}$ laser pulses use the same output mirror, thus ensuring that the pulses have the same direction of propagation. Both pulses are generated in the same diode pumped laser rod, ensuring that the pulses are collinear. Both pulses use the same Q-switch ensuring the same pulse initiation time. The 4 quartz prisms also separate the 2 laser beams, conveniently turning them $180^{\circ}$, and polarizing the laser beams. Highly reflecting mirrors at 1.064 and $1.319 \mu \mathrm{m}$ provide independent length control for the resonators.

Given the same initiation time of the 1.052 and $1.319 \mu \mathrm{m}$ laser pulses, synchronous pulses can be obtained by making the pulse evolution time intervals identical for the 2 pulses. Usually, this does not occur because the gain at the dominant wavelength is larger than the competing wavelengths. Because both transitions originate from the same upper manifold, the pulse that develops first depletes the population inversion before the competing pulses extracts a significant amount of energy. To compensate, the losses and resonator lengths can be adjusted so that a second pulse can successfully compete. The 1.052 and $1.319 \mu \mathrm{m}$ transitions are a good choice because they have similar emission cross sections, allowing for nearly equal resonator lengths.

A dual wavelength amplifier was developed to increase the utility of the dual wavelength laser oscillator. A twin stage Nd:YAG amplifier was built and evaluated on individual transitions and on 2 transitions simultaneously. [2] The dual wavelength pulses were amplified to increase the energy available for the sum frequency mixing and subsequent second harmonic generation. In addition a set of cylindrical lenses formed a telescope for focusing to increase the efficiency of the nonlinear processes.

A BBO crystal, $10 \mathrm{~mm}$ long, provided sum frequency mixing of the dual wavelength pulses, producing pulses at $0.585 \mu \mathrm{m}$. Since the fundamental pulses are collinear and synchronous, the sum frequency generation becomes as simple as second harmonic generation. Finally, a half wave plate at $0.59 \mu \mathrm{m}$ rotated the polarization $90^{\circ}$ degrees and a BBO crystal, $8 \mathrm{~mm}$ long provided second harmonic generation of the mixed signal, producing pulses at $0.293 \mu \mathrm{m}$. Harmonic generation on the individual transitions was also performed, producing 0.660 and $0.330 \mu \mathrm{m}$ from the $1.319 \mu \mathrm{m}$ transition and 0.526 and $0.266 \mu \mathrm{m}$ from the $1.052 \mu \mathrm{m}$ transition. 


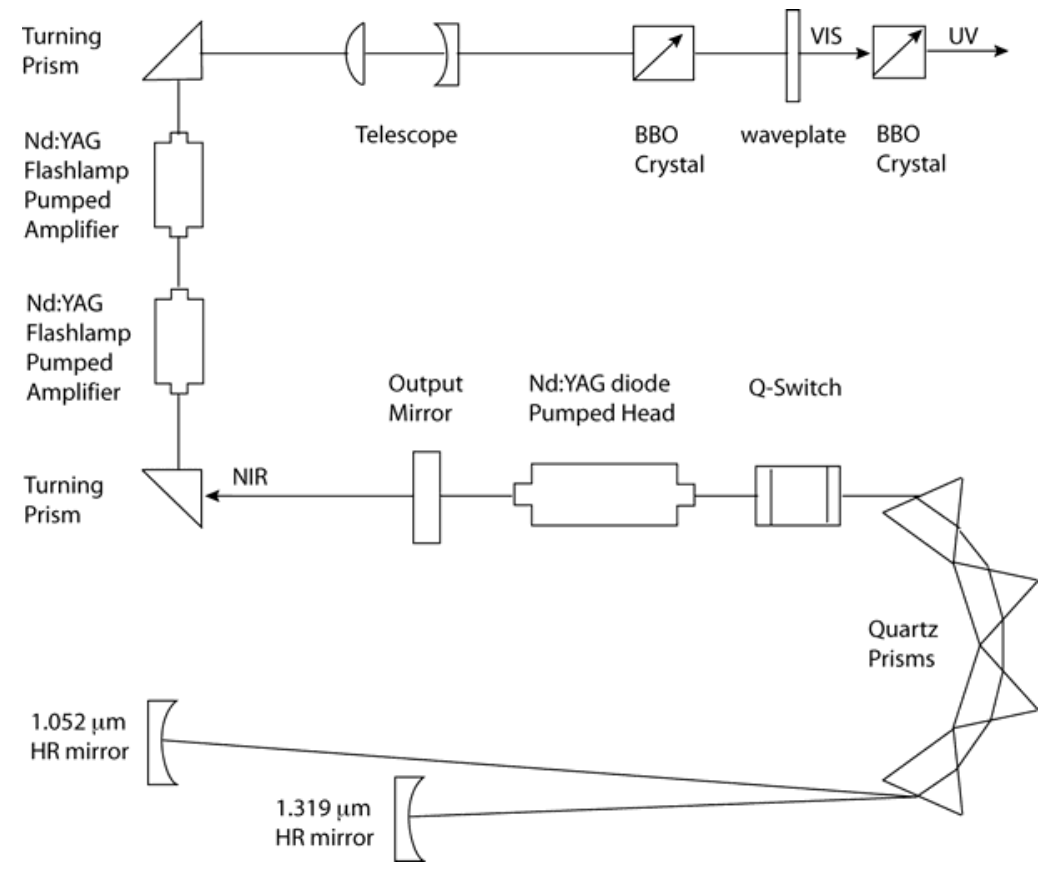

Figure 1. Schematic of ultraviolet laser using a dual wavelength Nd:YAG oscillator, 2 stage amplifier, sum frequency mixing and second harmonic generation.

\section{Results}

The 1.052 and $1.319 \mu \mathrm{m}$ pulses of the STOP laser can be mixed or doubled producing red, orange and green pulses at $0.660,0.585$, and $0.526 \mu \mathrm{m}$, respectively. The results are shown in figure 2 . In each case, a maximum energy of approximately $20 \mathrm{~mJ}$ was obtained. The maximum efficiencies were $0.21,0.16$ and 0.25 for the 0.660 , 0.585 , and $0.526 \mu \mathrm{m}$, respectively. The visible wavelengths were subsequently doubled producing ultraviolet light at $0.330,0.293$ and $0.263 \mathrm{~mm}$. The results are shown in figure 3. The efficiencies of the 0.293 and $0.263 \mu \mathrm{m}$ lasers were approximately 0.25 , while the $0.330 \mu \mathrm{m}$ efficiency was about 0.07 . The reason for the low efficiency on this second harmonic wavelength is due to the fact that a half waveplate at $0.660 \mu \mathrm{m}$ was not available. The BBO crystal had to be oriented for rotation in the horizontal instead of in the vertical, but this is not the desired arrangement to take advantage of the cylindrical focusing.

The versatility of this laser allows many more ultraviolet wavelengths than were measured here. The STOP laser can be tuned to 12 transition lines in the near infrared, 4 in the ${ }^{4} \mathrm{~F}_{3 / 2} \rightarrow{ }^{4} \mathrm{I}_{13 / 2}$ transition around $1.3 \mu \mathrm{m}$ and 8 in the ${ }^{4} \mathrm{~F}_{3 / 2} \rightarrow{ }^{4} \mathrm{I}_{11 / 2}$ transition around $1.06 \mu \mathrm{m}$. [3] This leads to a large number of possible wavelengths in the visible, ranging from 0.526 to $0.678 \mu \mathrm{m}$, and subsequently in the ultraviolet, ranging from 0.263 to $0.339 \mu \mathrm{m}$. With a properly designed resonator, any combination of the 44 possible laser transitions can be operated. The entire frequency conversion system becomes a compact, linear arrangement of 2 to 4 nonlinear crystals, and perhaps some lenses and waveplates. Since the STOP pulses are collinear and synchronous, the sum frequency mixing becomes as simple as simple harmonic generation. The optical efficiency of the laser from near IR fundamental to the ultraviolet is approximately 0.05 . Operation at higher oscillator energies, the use of diode pumped amplifiers, and better nonlinear conversion is expected to raise the efficiency. The simplicity of the design makes this laser particularly easy to modify and adapt for a tunable source in the near IR, visible and ultraviolet in one compact laser system. 


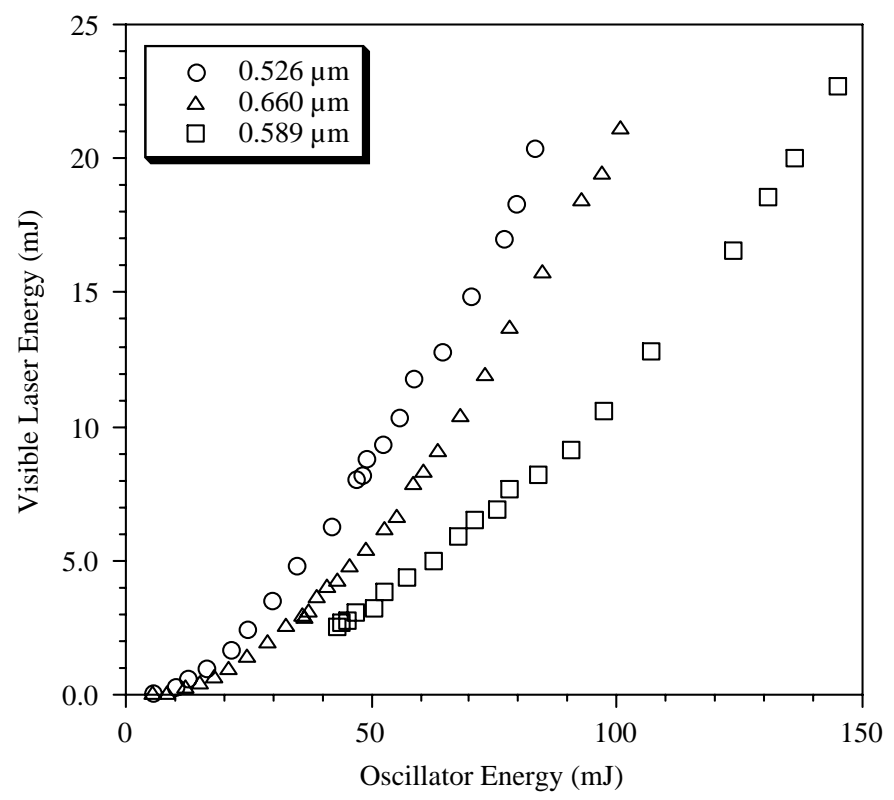

Figure 2. Second harmonic and sum frequency mixing laser energy versus oscillator energy

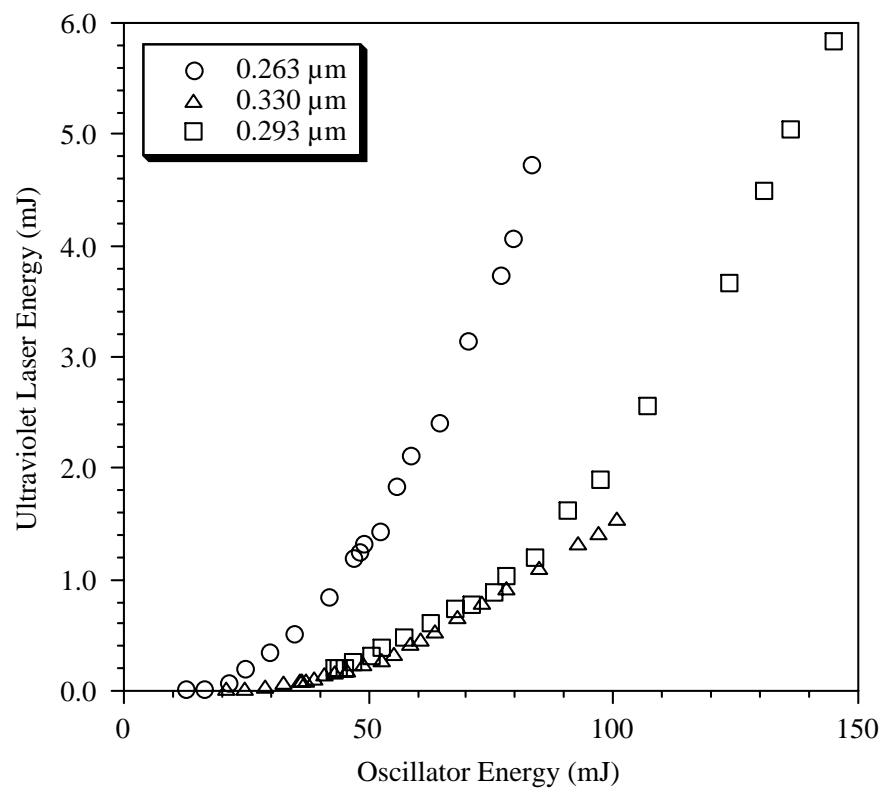

Figure 3. Second harmonic of visible laser energy versus oscillator energy

\section{References}

[1] N.P. Barnes and B.M. Walsh, "Synchronous Tunable Optical Pulses,” OSA TOPS, vol. 94, (2004).

[2] N.P. Barnes and B.M. Walsh, "Dual Wavelength Laser Amplifier,” OSA TOPS, this volume, (2005).

[3] N.P. Barnes and B.M. Walsh, Richard E. Davis “Dispersive Tuning and Performance of a Pulsed Nd:YAG Laser,” OSA TOPS, vol. 33, p. 38, (2004). 